\title{
Nanometrology and its perspectives in environmental research
}

\author{
Hyun-A Kim ${ }^{1}$, Jung-Kwan Seo ${ }^{2}$, Taksoo Kim², Byung-Tae Lee ${ }^{1}$ \\ ${ }^{1}$ School of Environmental Science and Engineering, Gwangju Institute of Science and Technology (GIST), Gwangju; \\ ${ }^{2}$ Division of Risk Assessment, National Institute of Environmental Research (NIER), Incheon, Korea
}

\begin{abstract}
Objectives Rapid increase in engineered nanoparticles (ENPs) in many goods has raised significant concern about their environmental safety. Proper methodologies are therefore needed to conduct toxicity and exposure assessment of nanoparticles in the environment. This study reviews several analytical techniques for nanoparticles and summarizes their principles, advantages and disadvantages, reviews the state of the art, and offers the perspectives of nanometrology in relation to ENP studies.

Methods Nanometrology is divided into five techniques with regard to the instrumental principle: microscopy, light scattering, spectroscopy, separation, and single particle inductively coupled plasma-mass spectrometry.

Results Each analytical method has its own drawbacks, such as detection limit, ability to quantify or qualify ENPs, and matrix effects. More than two different analytical methods should be used to better characterize ENPs.

Conclusions In characterizing ENPs, the researchers should understand the nanometrology and its demerits, as well as its merits, to properly interpret their experimental results. Challenges lie in the nanometrology and pretreatment of ENPs from various matrices; in the extraction without dissolution or aggregation, and concentration of ENPs to satisfy the instrumental detection limit.
\end{abstract}

Keywords Engineered nanoparticle, Light scattering, Microscopic analysis, Nanometrology, Separation technique, Single particle inductively coupled plasma-mass spectrometry

\author{
Correspondence: \\ Byung-Tae Lee, PhD \\ 123 Cheomdangwagi-ro, Buk-gu, \\ Cwangju 500-712, Korea \\ Tel: $+82-62-715-3315$ \\ Fax: $+82-62-715-3314$ \\ E-mail: btlee@gist.ac.kr \\ Received: July 28, 2014 \\ Accepted: September 19, 2014 \\ Published online: October 15, 2014 \\ This article is available from: $h$ ttp://e-eht.org/
}

\section{Introduction}

Owing to their high functionality, engineered nanoparticles (ENPs) are widely used in many industries as well as consumer products. The rapid increase in their use in many goods, including cosmetics and foods has raised significant concern about their environmental safety, especially with regards to their potential toxicity to humans and ecosystems $[1,2]$. Many studies have reported that ENPs have distinctive hazards to human or ecosystems from their composition [3]. Cell viability assays using A549 lung cells revealed that silver nanoparticle (AgNP) suspensions were more toxic when the initial silver ion fraction was higher [3]. The three most photosensitive common ENPs, titanium dioxide $\left(\mathrm{TiO}_{2}\right)$, silicon dioixde $\left(\mathrm{SiO}_{2}\right)$, and zinc oxide $(\mathrm{ZnO})$ were also found to be toxic to Escherichia coli by promoting generation of reactive oxygen species when exposed to light [4]. More serious is that ENPs persist in, or penetrate to places in the environment or organisms, thereby causing hazards [5].

The increase in the use of ENPs in many products has accelerated their release into the environment through the product lifecycle [6]. As AgNPs, due to the antibacterial effects, are the fastest growing category of ENPs for products concerns about their stability and hazards have also been increasing exponentially 
[7]. Several studies have demonstrated that AgNPs were released from sock fabrics and clothing during washing [8]. ENPs released in water environment inevitably go through various water chemistry, such as $\mathrm{pH}$, ionic strength, and natural organic matter, and their fate and transport are highly dependent on the given environmental conditions $[9,10]$. The aggregation rate of citrated-AgNPs increased with increasing ionic strength and decreasing natural organic matter (NOM) concentrations and the adsorption to soils is closely associated with environmental factors, such as ionic strength and NOM [10]. Aggregation of electrostatically stable ENPs is dominantly controlled by ionic strength, due to the change of electrostatic double layer, which is more compressed by high ionic strength resulting in an increasing van der Waals attraction [9]. Toxic metals released from ENPs have been found to play a significant role in toxicity and the dissolution of metals is also influenced by their environmental chemistry [11]. The dissolution rate of $\mathrm{Ag}$ from AgNP was highly affected by the concentration of chloride and sulfide and dissolved organic carbon significantly decreased the release of Ag from AgNP over 24 hours. Life cycle assessment of ENPs also showed that significant amounts of $\mathrm{Ag}$ were released from AgNP containing textiles by sweat and detergent solutions [12]. The surface area of ENPs also plays a significant role in their dissolution, in which smaller ENPs released metals more quickly [13]. Usually ENPs have polymer coating for the preservation and handling and different coating materials show distinctive surface properties, such as hydrophobicity and surface charges. Surface coatings have altered the toxicity of ENPs [14] and decreased the released metals [11].

The sound and sustainable development of nanotechnology requires risk assessment of ENPs. While traditional analysis has shown the total metal concentration in environmental samples by acid digestion, this method is not capable of suggesting particle information, which is critical to ENP risk assessment. Therefore, proper methodologies are needed to conduct toxicity and exposure assessment. Measurement of nanomaterials, which is called nanometrology should provide not only the concentration but also their size and size distribution because their toxicity and fate has been reported to rely on their size, specific surface area, and number concentration. Though metrologic and analytic methods are generally considered to be the most important for ENP risk assessment, it is inevitable that our state of knowledge on nanometrology is very low. Several conventional analytic tools have been used to characterize or analyze ENPs for their size, size distribution, and surface properties. In order to better understand the limitation of the studies that discussed the ENP toxicity or fate, to select the most proper analytic methods, and to improve experimental techniques, it is essential to know their principles, advantages and disadvantages, to review the state of the art, and to offer the perspectives of nanometrology on ENP studies.

\section{Engineered Nanoparticles and Characterization}

The most important properties of ENPs that should be characterized for environmental and ecotoxicology study are the size and number based size distribution. Nanomaterials have one dimension less than $100 \mathrm{~nm}$, whereas nano-objects have two dimensions less than $100 \mathrm{~nm}$, while nanoparticles have three dimensions of less than $100 \mathrm{~nm}$ [15]. The European Commission (EC) Framework Programme 7 has funded project 'NanoImpactNet', with an open forum for the discussion of current opinions on nanomaterials in relation to human and environmental issues. One of the issues in the 2008 workshop was "what properties should be characterized for nanomaterials". Six main properties of ENPs (size, dissolution, surface area, surface charge, surface composition, and surface chemistry) were chosen for the main priorities required for environmental and ecotoxicological studies. Among them, size and composition are the key properties because previous studies have shown that size played a significant role in the toxicity and ENPs act as a sink source of toxic metals. The EC has defined 'Nanomaterial' from a regulation aspect to natural, incidental, or manufactured materials containing particles, in an unbound state or as an aggregate or an agglomerate, and where, for $50 \%$ or more of the particles in number size distribution, one or more external dimensions is in the size range 1-100 $\mathrm{nm}$ [16]. This recommendation is solely based on the size of the constituent particles and refers to the number based size distribution.

\section{Microscopic Analysis}

Several powerful microscopic analyses can provide not only images of ENPs but also information on their elemental compositions. These techniques are based on visualizing ENPs using light, electron, or scanning probe. Among them, electron microscopy techniques are most common to detect and size ENPs, which have sub-nm resolution. Transmission electron microscopy (TEM) uses a bean of electrons that are transmitted through an ultra-thin specimen, interacting with a specimen as it passes through. From the interaction of the electrons transmitted through the specimen, an image is formed, magnified, and focused onto an imaging device, such as a fluorescent screen or a charge-coupled device camera (Figure 1). The resolution of TEM reaches about $0.07 \mathrm{~nm}$, which is highly dependent on the 

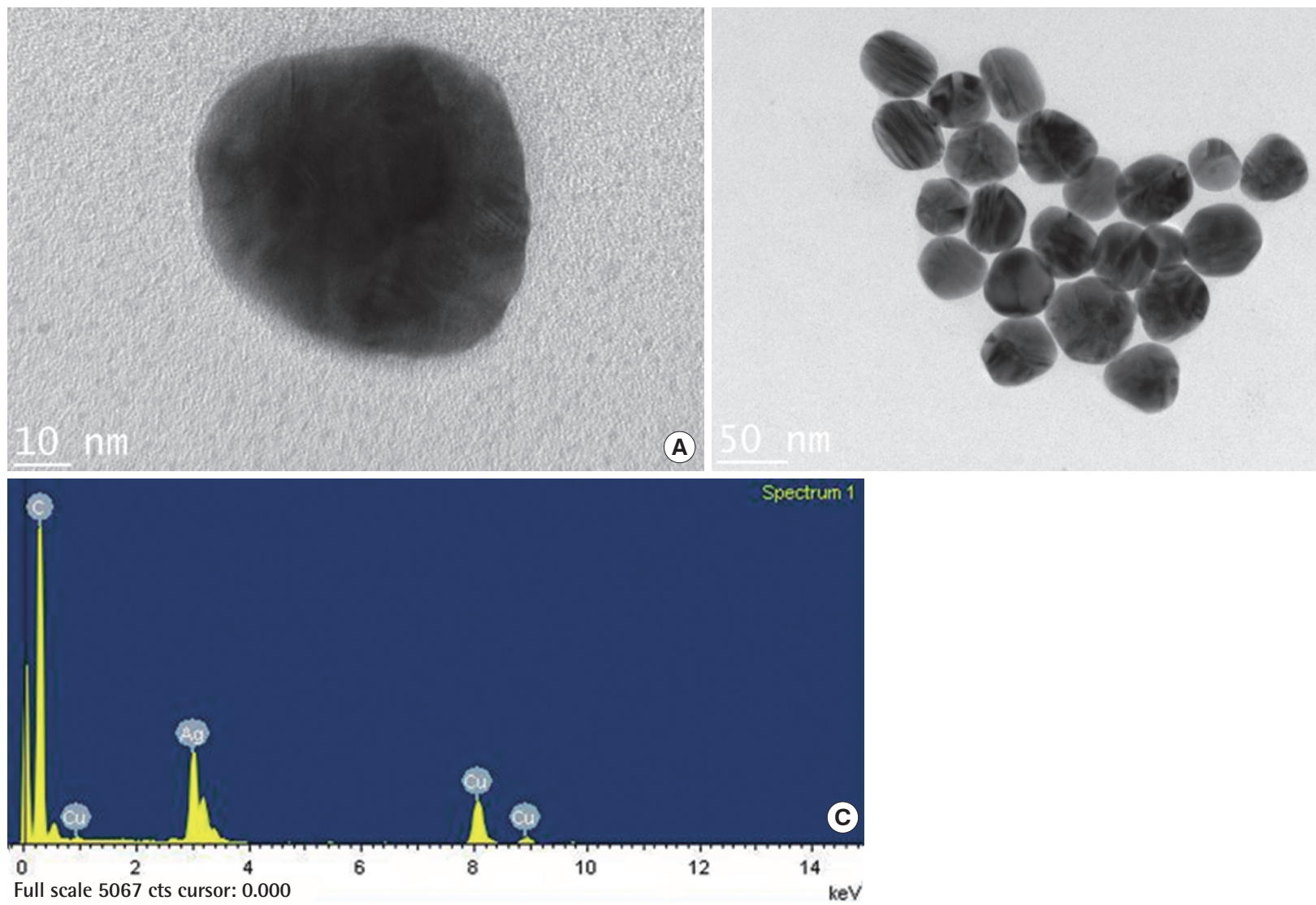

Figure 1. Trasnsmission electronmicroscopy images (A, B) and elemental composition analysis (C) by energy dispersed X-ray spectroscopy on $60 \mathrm{~nm}$ citrate coated silver nanoparticle (NanoComposix, USA).

thickness of the prepared sample as well as the accelerating voltage for the electron beam. Scanning electron microscopy (SEM) is another representative electron microscopic technique. Electron beam of SEM is generally scanned in a rater scan pattern and the beam position is combined with detected signal to produce an image. SEM has less resolving power than TEM. High resolution SEM has achieved resolutions of $1 \mathrm{~nm}$ and conventional SEM can detect up to $3 \mathrm{~nm}$ [17]. One advantage of SEM is that environmental SEM allows sample imaging under low pressure, fairly high humidity and without the requirement of a conducting overcoat [18]. Besides imaging ENPs, elemental composition can be determined using energy dispersive X-ray spectroscopy (EDS or EDX).

The most critical issue in electron microscopy (EM) analysis is sample preparation. It is very challenging to obtain representative shape, size, and size distribution results from a complex bulk structure because the sample volume analyzed by EM is very small. If the concentration of ENPs in the samples is not high enough, the sample should be pre-concentrated by cloud point extraction or centrifugation [19]. Recently, the National Institute of Standard and Technology (NIST) provided the standard protocol to size ENPs using TEM and SEM [20, 21]. PCC-7 works with ENPs that carry a negative surface charge or zeta potential. Briefly, TEM grid is derivatized by exposure to a small amount of amino propyl-dimethyl-ethoxy silane (APDMES, $\left.\mathrm{NH}_{2}-\left(\mathrm{CH}_{2}\right)_{3}-\mathrm{Si}-\left(\mathrm{CH}_{3}\right)_{2}\left(\mathrm{C}_{2} \mathrm{H}_{5} \mathrm{O}\right)\right)$ solution which serves to attach well-separated positive charges and attract negatively charged ENPs. To get a representative size and size distribution, a minimum of 200 discrete particles is recommended to be measured from each of at least two widely separated regions of the sample. Sample preparation protocol for SEM by PCC-15 also has similar procedures using APDMES. As the distinctive feature of SEM is that SEM images are essentially two-dimensional projections of the non-spherical particles, evaluating the results from both EMs is usually recommended to characterize ENPs on shape and size. Plathe et al. [22] provided a sample preparation method for TEM, which uses resin mixture $(1.0 \mathrm{~g}$ melamine resin $+0.025 \mathrm{~g}$ catalyst). The resin is used to prevent artifacts from forming during evaporation of the sample. After pipetting the sample mixture, the grid is centrifuged fast enough to produce a very thin veneer of resinated samples and dried in an oven. 


\section{Light Scattering}

Light scattering methods use light as propagating energy which is scattered by target analytes. The interaction of light with the target generates important information about the structure and dynamics of the target being examined. Commonly, light scattering terminology is divided into static light scattering (SLS) and dynamic light scattering (DLS). The DLS technique is usually applied to measure ENP sizes (hydrodynamic size or z-average), which is an intensity-weighted average. DLS is also known as photon correlation spectroscopy and is noninvasive, nondestructive, and relatively cheap [18].

When light hits ENPs, the light scatters in all directions which is Rayleigh scattering in the case that the ENPs are small compared to the wavelength $(<250 \mathrm{~nm})$. Usually, the light source is a laser, which is monochromatic and coherent. As ENPs undergo Brownian motion in suspension, the scattering intensity fluctuates over time constructing an autocorrelation function from the measured fluctuations in the scattered light intensity over time. The autocorrelation function is transformed to a function of the particle diffusion coefficient from which the equivalent hydrodynamic diameter of particles is determined using the Stokes-Einstein relationship with the assumption that particles are spherical and hard (nonpermeable) [23].

DLS can provide two analysis methods for autocorrelation function. Cumulant analysis is used to determine the z-average hydrodynamic diameter and polydispersity index (PDI), while distribution analysis gives intensity weighted size distribution. DLS has several advantages: operation is relatively simple and rapid, the instrument is available, and there is little perturbation of the sample. This method has a broad size range of 3->1,000 $\mathrm{nm}$ but its limit of detection is at the $\mathrm{mg} / \mathrm{L}$ level [24]. The critical disadvantage is that the data interpretation is problematic for polydispersed systems. The scattering intensity strongly depends on the particle size (Figure 2). The $30 \mathrm{~nm}$ particle shows $10^{6}$ times stronger scattering intensity than $3 \mathrm{~nm}$ particle resulting in the error that small particle are neglected. Therefore, a small amount of dust or larger particles can wreck the data interpretation of DLS and its reliability. When ENPs are analyzed for their hydrodynamic size by DLS, the sample should be checked for whether the PDI is low enough to be considered as monodispersed.

In most studies of ENP fate and transport, DLS has been widely used to record the aggregation of particles over time [10]. Different $\mathrm{pH}$, ionic strength (IS), and NOM are controlled, to better understand the effect of aqueous chemistry on the fate of ENPs in the environment in terms of aggregation by calculating the aggregation kinetic rate. Though this experimen-
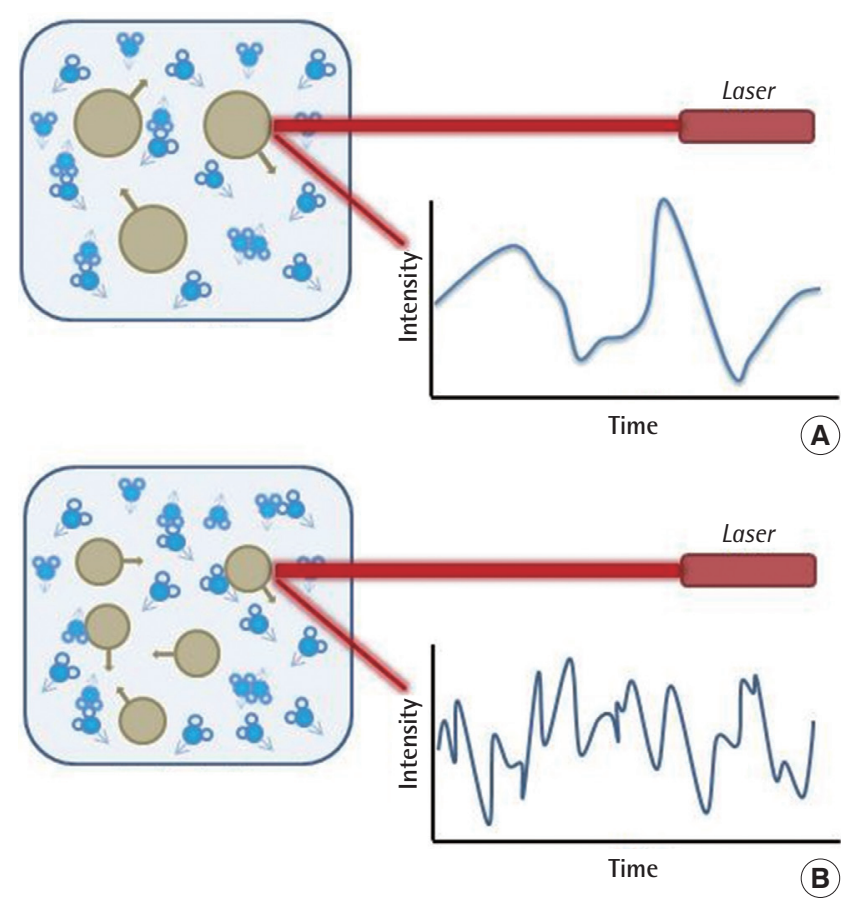

Figure 2. Hypothetical fluctuation of scattered light in dynamic light scattering from (A) large and (B) small particles. As larger particles generates higher scattering intensity than smaller particles [25].

tal approach has successfully explained the role of water chemistry, the particle concentration in the measurement was much higher (usually $>1 \mathrm{mg} / \mathrm{L}$ in the case of AgNP) than its predicted environmental concentration (below $1 \mu \mathrm{g} / \mathrm{L}$ ) [5]. Particle aggregation is dependent on both the frequency of particle collision and the attachment efficiency. The former is a function of particle concentration and the latter is highly dependent on particle properties, such as its surface charge and surrounding chemistry ( $\mathrm{pH}, \mathrm{IS}$, and NOM). As experiments using DLS are far away from ENPs environmental concentration, the aggregation kinetics from DLS can explain only the qualitative aspect. Another limitation of DLS is that it is not capable of determining the aggregation state in samples even though it can be applied to micro-sized particles. In aggregation study, increasing particle size over time also increases the PDI. As mentioned earlier, high PDI does not provide precise size of particles in samples. Therefore, the $\mathrm{z}$-average of aggregates is not reliable. A practical tip for DLS measurement is to prevent samples from unintended dust or macro particles. The cuvette or vials for DLS should be cleaned several times by flowing deionized (DI) water and all solutions should be prepared using DI water that is pre-filtered by $0.1 \mu \mathrm{m}$ membrane.

\section{Spectroscopic Techniques}

This technique utilizes the interaction of electromagnetic radia- 
tion with a sample material exhibiting the absorbance at UV-Vis or emission at fluorescence. Spectroscopic analysis is commonly used to characterize the bulk samples. The spectrum shows the absorbance dependency on the wavelength. While the absorbance is a quantitative signal, the wavelength is qualitative. To quantify the samples using the absorbance or fluorescence, the optical constants of the particles should be known. UV-Vis spectroscopy is the representative technique which uses the absorbance of the sample at specific wavelength. It has been widely used to measure concentrations, size, and aggregation state. Though it has been assumed that $\mathrm{UV}$ absorbance is proportional to ENP concentrations, it could be affected by the wavelength of the light, the size of the particle, and the matrix composition. Our previous study used UV absorbance to characterize the initial size and aggregation state of ENPs in synthetic fresh waters and the disappearance rate of monodispersed AuNPs was estimated using absorbance [9]. Qualitative information was also provided by explaining the peak wavelength shift during experiment. As higher wavelength for maximum absorbance occurs at larger ENPs, the red shift of wavelength could be interpreted into the formation and growth of aggregate.

Fluorescence is the light emission produced by an electron returning from a singlet excited state to a singlet ground state. When ENPs (for example, CdSe/ZnS quantum dot) are irradiated with light of a certain excitation wavelength, strong light is emitted at a certain emission wavelength. This combination of two wavelengths provides the fluorescence detection of ENPs. Quantitative analysis can be performed in the case that the optical constants of ENPs are known, for example extinction coefficient. Experimentally, the extinction coefficients of the first excitonic absorbance peak, $\varepsilon$, were determined and strongly depended on the quantum dot size [26]. The most critical limitation of spectroscopy in ENP analysis is that the background absorption is hard to distinguish from the target samples. To apply this to environmental samples, the background should be clearly determined prior to sample analysis and proven not to affect the sample spectrum (absorbance or emission).

The UV absorbance and fluorescence have been connected to separation techniques, such as chromatography and field flow fractionation (FFF) as online detector. The two spectroscopic analyses are distinctively different in their sensitivity to ENP concentration; fluorescence has usually much higher sensitivity than UV-Vis (Figure 3). Therefore, UV-Vis analysis is operated from $\mu \mathrm{g} / \mathrm{L}$ to $\mathrm{mg} / \mathrm{L}$, while fluorescence can be used for below $\mu \mathrm{g} / \mathrm{L}$. Spectroscopic techniques only provide the relative concentrations and need to be calibrated for quantitative analysis using standard materials.

\section{Separation Techniques}

As the separation techniques are relatively easy and cheap, they are widely applied for particle experiments. Filtration is the most general method. Microfiltration is used to remove any
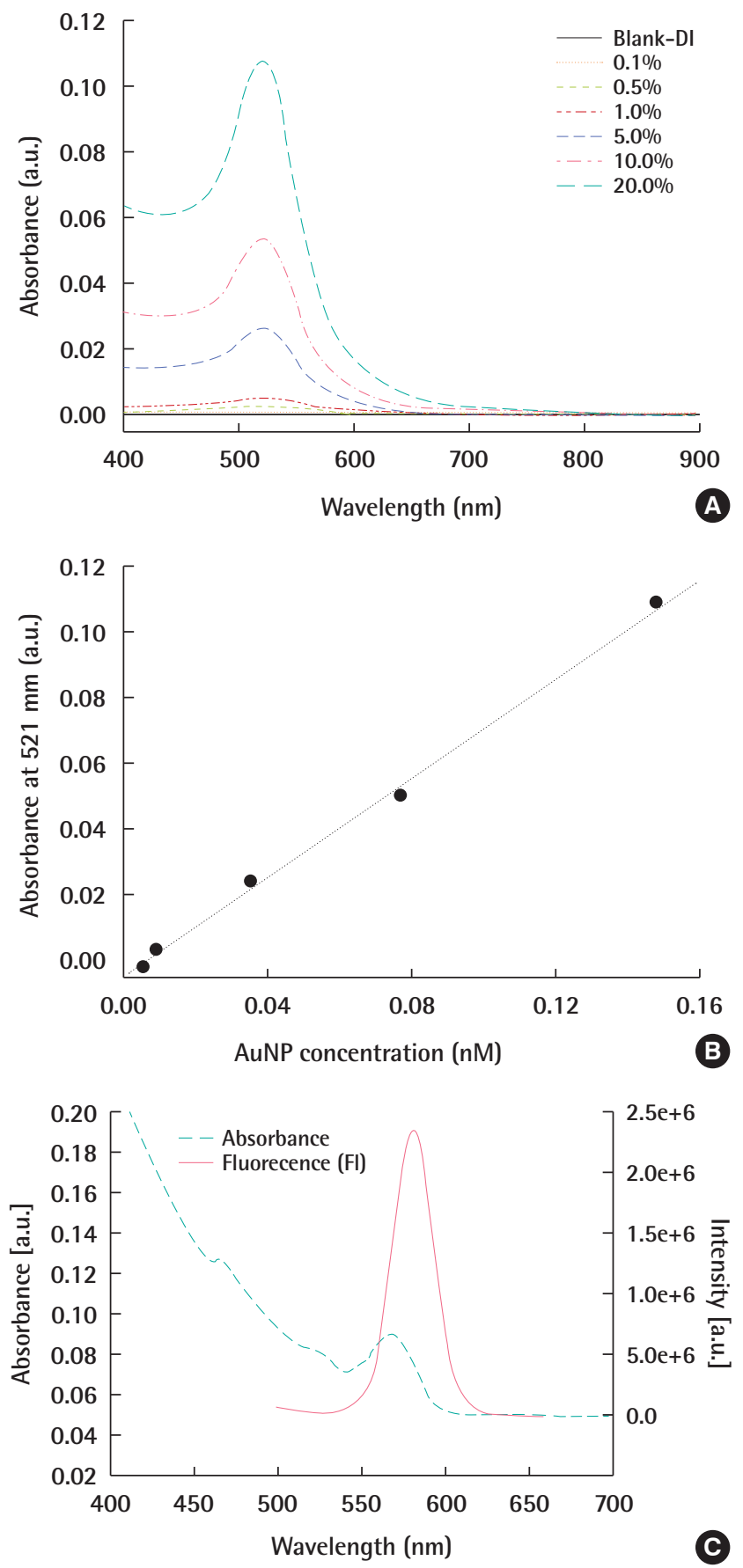

Figure 3. UV-Vis spectra of serally diluted $17 \mathrm{~nm}$ citrate coated gold nanoparticles in deionized (DI) water. The stock suspension contains 0.745 $\mathrm{nm}$ of AuNPs. At $521 \mathrm{~nm}$, (A) the spectra shows the maximum absorptions which shows, (B) linear relationship with AuNP concentration, and (C) CdSe/ ZnS quantum dot (QD) showed the distinctive UV-Vis spectra and FI. The FI intensity is much higher than UV-Vis absorbance even though the QD concentration is 100 times lower in Fl measurement. 
macro particles from micro or nanoparticles. The pore size of microfiltration is greater than $0.1 \mu \mathrm{m}$. Even though the pore size is larger than the nanoparticles, many artifacts exist, for example, deposition, membrane concentration polarization, and filter cake formation [27]. ENPs can be deposited on the membrane surface due to collision or electrostatic attraction [28]. In the case that ENPs have electrostatic repulsion to the membrane, ENPs can build up of higher particle concentration. This phenomenon is called concentration polarization resulting in the higher collision rates. The formation of aggregation or trapping macroparticles decreases the pore size leading to the formation of filter cake and significantly decrease the effective pore size. The composition of the filter membrane also produces artifacts in the separation. Nylon severely retained $20 \mathrm{~nm}$ AgNPs even with $0.45 \mu \mathrm{m}$ pore size [29]. Therefore, filtration for ENPs should be carefully evaluated for ENP recovery. Few studies have used the chromatographic separation for carbon nanoparticles. High performance liquid chromatography with UV-Vis spectrometry at $350 \mathrm{~nm}$ has been used to analyze fullerene [30]. Gel permeation chromatography and size exclusion chromatography have also been used for carbon nanoparticles or ENPs $[31,32]$. Cloud-point extraction uses surfactants, such as Triton X-114 (TX-114) to form micellar structure [19]. After the surfactant is added into the sample at a concentration that exceeds the critical micellar concentration, the surfactant forms micelles that trap nonpolar substances, such as ENPs. As these micelles have higher density than water, they can be separated by sedimentation or centrifugation.

Filed-flow fractionation (FFF) is one of the promising separation techniques for nanoparticle analysis. It is a chromatographylike size fractionation method that uses field flow and cross flow instead of stationary phase. The most common FFF type is flow FFF (FIFFF) that separates particles by their diffusion coefficient in a very thin open channel [33]. The key feature of FIFFF is that the size range is $1-1,000 \mathrm{~nm}$, even though the detection limit is highly dependent on the detector coupling to FIFFF for example UV (ppm), fluorescence (ppb), or inductively coupled plasma mass spectrometry (ICP-MS, ppt-ppb) [24]. Because ENPs in samples are laid on an accumulation wall that is usually a membrane, there is attractive or repulsive interaction. When the attractive interaction is strong enough to retain samples, the particles flow slowly or are concentrated on the wall. In contrary, significant repulsion can lead to unexpected early elution of particles, leading to underestimation of their size. Therefore, FlFFF should determine the optimal conditions of carrier solution and membrane before separation. The FIFFF has been successfully applied to characterize ENPs from food [2] and environmental samples [22], and organic nanoparticles [34].

\section{Single Particle Inductively Coupled Plasma-mass Spectrometry}

Single particle ICP-MS (spICP-MS) is a promising technique that has the potential to determine particle size, number concentration, and aggregation state in an environmentally relevant concentration. The basic theory of spICP-MS has been explained by Degueldre et al. [35, 36], who have provided several equations for calculating sizes from the counts of ICP-MS. Traditional ICP-MS integrates intensity over a long reading time ( $>300 \mathrm{~ms}$ ), to calculate the metal concentrations in samples. The spICP-MS uses a relatively short reading time (usually less than $10 \mathrm{~ms}$ ) which is called dwell time in realtime mode, and produces the individual counts per time (count per second or count per dwell time). Dissolved metal produces continuous and constant signals with time, while a particle in samples generates a discontinuous ion cloud at the plasma resulting in a pulse signal. The spICP-MS has two basic assumptions: that each pulse signal represents one particle and the signal intensity corresponds to the mass of element in the particle. The procedures of size calculation from instrumental signals are shown in a previous study [37]. Briefly, the first step is to convert calibration information of count vs. concentration to count vs. mass by considering sample flow rate, dwell time, and transport efficiency (nebulizer efficiency). In the next step, signals are sorted by frequency and are divided into background and particle signals. Each particle signal is further treated to calculate mass from its count using the calibration information and the mass is finally converted into the equivalent spherical diameter.

This method has been successfully applied to analyze ENPs such as $\mathrm{AgNP}, \mathrm{AuNP}$, and $\mathrm{TiO}_{2}$ and the data processing has been well established [37]. Our study also proved this technique is capable of analyzing ENPs and the result corresponds to the size or size distribution by TEM (Figure 4). As individual particles should be introduced into plasma, spICP-MS requires highly diluted samples that are diluted enough to avoid coincidence and obtain single particle counts per dwell time. However, this limitation has a critical advantage in analyzing ENPs because ENP concentration in the environment is predicted to be below $\mathrm{ppb}$. Sample containing polydispersed ENPs was analyzed by spICPMS and was well distinguished from coincidence suggesting that it can be used to determine the ENP aggregation [38]. In addition, spICP-MS detected ENPs in natural water samples and characterized the dissolution of ENPs with time [11]. In 2003, spICP-MS were proposed to the International Organization for Standardization and is under review as a standard method to detect and characterize nanoparticles [39]. 

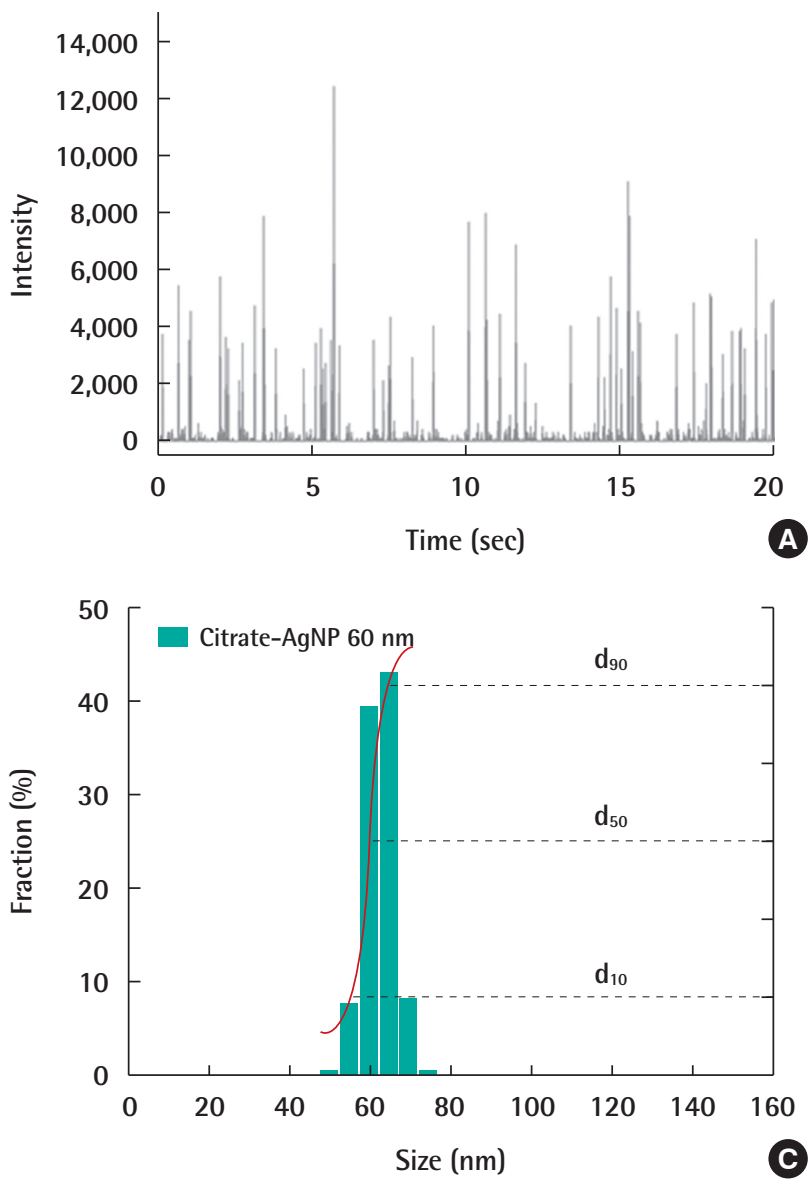

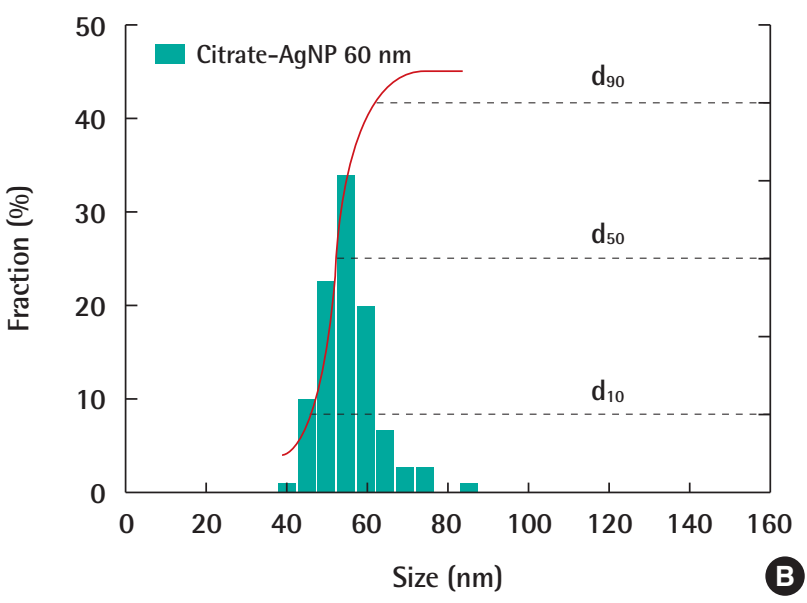

Figure 4. Analytical results of $60 \mathrm{~nm}$ citrate-AgNP (nanoComposix) suspensions; (A) time-series signals from inductively coupled plasma mass spectrometry (ICP-MS) (Elan DRC II, PerkinElmer) at realtime mode with $10 \mathrm{~ms}$ dwell time, (B) differential size distribution and cumulative undersize by splCP-MS, and (C) comparable size distributions of transmission electron microscopy analysis.

Table 1. Characterization and specification of analytical methods for nanoparticles

\begin{tabular}{|c|c|c|c|c|c|c|c|c|c|}
\hline Method & Measurement & Advantages & Limitations & $\begin{array}{c}\text { Size } \\
\text { range } \\
(\mathrm{nm})\end{array}$ & $\begin{array}{c}\text { Required } \\
\text { concentration }\end{array}$ & $\begin{array}{l}\text { Single } \\
\text { particle }\end{array}$ & Quantify & Qualify & $\begin{array}{l}\text { Matrix } \\
\text { effect }\end{array}$ \\
\hline $\begin{array}{l}\text { Scanning electron } \\
\text { microscopy }\end{array}$ & $\begin{array}{l}\text { Size } \\
\text { Morphology } \\
\text { Composition }\end{array}$ & $\begin{array}{l}\text { High resolution } \\
\text { Determine } \\
\text { composition }\end{array}$ & $\begin{array}{l}\text { Dry samples needed } \\
\text { Need coating process }\end{array}$ & $>10$ & $\mathrm{ppb}$ & Single & $x$ & 0 (EDS) & Medium \\
\hline $\begin{array}{l}\text { Transmission } \\
\text { electron microscopy }\end{array}$ & $\begin{array}{l}\text { Size } \\
\text { Morphology }\end{array}$ & $\begin{array}{l}\text { Analyze individual } \\
\text { nanoparticle size } \\
\text { and shapes }\end{array}$ & $\begin{array}{l}\text { Dry samples needed } \\
\text { Sample damage by } \\
\text { ionizing radiation }\end{array}$ & $>1$ & ppb & Single & $x$ & 0 (EDS) & Medium \\
\hline $\begin{array}{l}\text { Dynamic light } \\
\text { scattering }\end{array}$ & $\begin{array}{l}\text { Hydrodynamic } \\
\text { diameter } \\
\text { Zeta potential }\end{array}$ & $\begin{array}{l}\text { Nondestructive } \\
\text { Rapid, simple }\end{array}$ & $\begin{array}{l}\text { Pretreatment needed } \\
\text { Limited ability for } \\
\text { poly-disperse samples }\end{array}$ & $>3$ & ppm & Bulk & $x$ & $x$ & High \\
\hline $\begin{array}{l}\text { UV-Visible } \\
\text { spectroscopy }\end{array}$ & $\begin{array}{l}\text { Concentration } \\
\text { Size } \\
\text { Aggregation state }\end{array}$ & $\begin{array}{l}\text { Cost effective } \\
\text { Nondestructive }\end{array}$ & Low sensitivity & - & ppm & Bulk & 0 & $x$ & High \\
\hline Fluorescence & Size & Sensitive & $\begin{array}{l}\text { Intrinsic or extrinsic } \\
\text { fluorescence needed }\end{array}$ & - & - & Bulk & 0 & $x$ & High \\
\hline $\begin{array}{l}\text { Flow filed-flow } \\
\text { fractionation }\end{array}$ & $\begin{array}{l}\text { Nanoparticle } \\
\text { separation } \\
\text { Size \& size } \\
\text { distribution }\end{array}$ & $\begin{array}{l}\text { High resolution } \\
\text { Applicability to wide } \\
\text { range of sizes }\end{array}$ & $\begin{array}{l}\text { Coupled detector } \\
\text { needed }\end{array}$ & $>1$ & ppt ppm & Bulk & $\begin{array}{c}0 \\
(\mathrm{ICP}-\mathrm{MS})\end{array}$ & $\begin{array}{c}0 \\
(\mathrm{ICP}-\mathrm{MS})\end{array}$ & High \\
\hline $\begin{array}{l}\text { Single particle } \\
\text { ICP-MS }\end{array}$ & $\begin{array}{l}\text { Size \& size } \\
\text { distribution } \\
\text { Number } \\
\text { concentration }\end{array}$ & $\begin{array}{l}\text { Low concentration } \\
\text { detection limit }\end{array}$ & Pretreatment needed & $>30$ & ppt & Single & 0 & 0 & Low \\
\hline
\end{tabular}

EDS, energy-dispersive spectroscopy; ICP-MS, inductively coupled plasma mass spectrometry. 


\section{Conclusion; Nanometrology and Its Perspectives}

Table 1 shows the brief characterization and specifications of various analytical methods for ENPs. Particle concentrations for instruments vary from ppt to ppm. The FlFFF highly depends on the detectors such as UV-Vis, Fl, DLS, and ICP-MS and is capable of detecting ppt level ENPs with Fl and/or ICP-MS. Even though spICP-MS has the highest sensitivity to metals, the size detection limit is not small enough to cover all ENP sizes. For AgNPs, the reported size detection limit is about $30 \mathrm{~nm}$ and is subject to the background signals. Recently, a shorter dwell time $(<100 \mu \mathrm{s})$ has been used to collect more data points for one pulse, so as to provide chromatography-like signals, which method is expected to obtain a more stable background and decrease the size detection limit. Instrumental improvement can lead to more precise and accurate analysis by spICP-MS.

The challenging study is the sample preparation. Both biological samples and environmental samples (water, sediment, and soils) consist of complex matrices, such as clay, naturally occurring metal oxides, organic debris, and inorganic salts. Traditional extraction methods for metal analysis use strong acids, leading to complete dissolution of metal particles including ENPs. The sample preparation has critical requirements: macro particles should be removed and the extraction should not dissolve ENPs and induce aggregation to analyze ENPs as it is in samples. Coarse filtration using $-1 \mu \mathrm{m}$ size filter paper can remove macro particles from samples, but this procedure should confirm fouling, deposition and attachment of ENPs. Mild extraction has been designed to set ENPs free from sample matrix using tetramethylammonium hydroxide and/or sonication [40]. Stabilizer, such as bovine serum albumin or surfactant (TX-114 or SDS) can be used to maintain ENPs stability after extraction. If the particle concentration in extracts is not high enough for instrumental analysis, a concentration step is needed. Though several attempts have been made to extract ENPs from various matries, few study provided their recovery. There is still a long way to go to achieve proper extraction methods as well as instrumental methods to analyze ENPs in various sample matrixes, including environmental samples and products containing ENPs. Only the precise and accurate characterization of ENPs will improve our understanding of the fate or transport, exposure, and even toxicity of ENPs. That is why the research should preferentially focus on nanometrology.

\section{Acknowledgements}

This research was supported by the National Institute of Envi- ronmental Research (NIER) and the National Research Foundation of Korea (2013R1A1A1007708).

\section{Conflict of Interest}

The authors have no conflicts of interest with material presented in this paper.

\section{References}

1. Weir A, Westerhoff P, Fabricius L, Hristovski K, von Goetz N. Titanium dioxide nanoparticles in food and personal care products. Environ Sci Technol 2012;46(4):2242-2250.

2. von der Kammer F, Legros S, Hofmann T, Larsen EH, Loeschner K. Separation and characterization of nanoparticles in complex food and environmental samples by field-flow fractionation. Trends Analyt Chem 2011;30(3):425-436.

3. Beer C, Foldbjerg R, Hayashi Y, Sutherland DS, Autrup H. Toxicity of silver nanoparticles - nanoparticle or silver ion? Toxicol Lett 2012; 208(3):286-292.

4. Adams LK, Lyon DY, Alvarez PJ. Comparative eco-toxicity of nanoscale $\mathrm{TiO} 2, \mathrm{SiO} 2$, and $\mathrm{ZnO}$ water suspensions. Water Res 2006; 40(19):3527-3532.

5. Gottschalk F, Sun T, Nowack B. Environmental concentrations of engineered nanomaterials: review of modeling and analytical studies. Environ Pollut 2013;181:287-300.

6. Nowack B, Ranville JF, Diamond S, Gallego-Urrea JA, Metcalfe C, Rose J, et al. Potential scenarios for nanomaterial release and subsequent alteration in the environment. Environ Toxicol Chem 2012; 31(1):50-59.

7. Maurer-Jones MA, Gunsolus IL, Murphy CJ, Haynes CL. Toxicity of engineered nanoparticles in the environment. Anal Chem 2013; 85(6):3036-3049.

8. Benn TM, Westerhoff P. Nanoparticle silver released into water from commercially available sock fabrics. Environ Sci Technol 2008;42(11):4133-4139.

9. Lee BT, Ranville JF. The effect of hardness on the stability of citrate-stabilized gold nanoparticles and their uptake by Daphnia magna. J Hazard Mater 2012;213-214:434-439.

10. Bae S, Hwang YS, Lee YJ, Lee SK. Effects of water chemistry on aggregation and soil adsorption of silver nanoparticles. Environ Health Toxicol 2013;28:e2013006.

11. Mitrano DM, Ranville JF, Bednar A, Kazor K, Heringd AS, Higgins CP. Tracking dissolution of silver nanoparticles at environmentally relevant concentrations in laboratory, natural, and processed waters using single particle ICP-MS (spICP-MS). Environ Sci Nano 2014; 1(3):248-259.

12. Hedberg J, Skoglund S, Karlsson ME, Wold S, Odnevall Wallinder I, Hedberg Y. Sequential studies of silver released from silver nanoparticles in aqueous media simulating sweat, laundry detergent solutions and surface water. Environ Sci Technol 2014; 48(13):7314-7322.

13. Dobias J, Bernier-Latmani R. Silver release from silver nanoparticles in natural waters. Environ Sci Technol 2013;47(9):4140-4146. 
14. Trujillo-Reyes J, Vilchis-Nestor AR, Majumdar S, Peralta-Videa JR, Gardea-Torresdey JL. Citric acid modifies surface properties of commercial $\mathrm{CeO} 2$ nanoparticles reducing their toxicity and cerium uptake in radish (Raphanus sativus) seedlings. J Hazard Mater 2013;263 Pt 2:677-684.

15. British Standards Institution. Terminology for nanomaterials, PAS 136: 2007 [cited 2014 Oct 10]. Available from: http://shop.bsigroup.com/upload/Shop/Download/Nano/PAS136.pdf.

16. European Commission. Commission recommendation (2011/ 696/EU) [cited 2014 Oct 10]. Available from: http://eur-lex.europa.eu/LexUriServ/LexUriServ.do?uri=OJ:L:2011:275:0038:00 40:EN:PDF.

17. Dudkiewicz A, Tiede K, Loeschner K, Jensen LS, Jensen E, Wierzbicki $\mathrm{R}$, et al. Characterization of nanomaterials in food by electron microscopy. Trends Analyt Chem 2011;30(1): 28-43.

18. Sapsford KE, Tyner KM, Dair BJ, Deschamps JR, Medintz IL. Analyzing nanomaterial bioconjugates: a review of current and emerging purification and characterization techniques. Anal Chem 2011;83(12):4453-4488.

19. Hartmann G, Hutterera C, Schuster M. Ultra-trace determination of silver nanoparticles in water samples using cloud point extraction and ETAAS. J Anal At Spectrom 2013;28(4):567-572.

20. National Institute of Standards and Technology. Measuring the size of nanoparticles using transmission electron microscopy (TEM); 2010 [cited 2014 Oct 10]. Available from: http://ncl.cancer.gov/ NCL_Method_PCC-7.pdf.

21. National Institute of Standards and Technology. Measuring the size of colloidal gold nano-particles using high-resolution scanning electron microscopy; 2011 [cited 2014 Oct 10]. Available from: http://ncl.cancer.gov/NCL_Method_PCC-15.pdf.

22. Plathe KL, von der Kammer F, Hassellöv M, Moore J, Murayama M, Hofmann T, et al. Using FlFFF and aTEM to determine trace metal-nanoparticle associations in riverbed sediment. Environ Chem 2010;7(1):82-93.

23. Baalousha M, Lead JR. Rationalizing nanomaterial sizes measured by atomic force microscopy, flow field-flow fractionation, and dynamic light scattering: sample preparation, polydispersity, and particle structure. Environ Sci Technol 2012;46(11):6134-6142.

24. Hassellöv M, Readman JW, Ranville JF, Tiede K. Nanoparticle analysis and characterization methodologies in environmental risk assessment of engineered nanoparticles. Ecotoxicology 2008; 17(5):344-361.

25. Li Y, Barron AR. Dynamic light scattering [cited 2014 Nov 24]. Available from: http://cnx.org/contents/3fc98dad-934d-45e6a19f-c0a1cf440d43@2.

26. Yu WW, Qu L, Guo W, Peng X. Experimental determination of the extinction coefficient of $\mathrm{CdTe}, \mathrm{CdSe}$, and CdS nanocrystals. Chem Mater 2003;15(14):2854-2860.
27. Morrison MA, Benoit G. Filtration artifacts caused by overloading membrane filters. Environ Sci Technol 2001;35(18):3774-3779.

28. Gimbert LJ, Haygarth PM, Beckett R, Worsfold PJ. Comparison of centrifugation and filtration techniques for the size fractionation of colloidal material in soil suspensions using sedimentation fieldflow fractionation. Environ Sci Technol 2005;39(6):1731-1735.

29. Mitrano DM, Lesher EK, Bednar A, Monserud J, Higgins CP, Ranville JF. Detecting nanoparticulate silver using single-particle inductively coupled plasma-mass spectrometry. Environ Toxicol Chem 2012;31(1):115-121.

30. Nowack B, Bucheli TD. Occurrence, behavior and effects of nanoparticles in the environment. Environ Pollut 2007;150(1):522.

31. Flavel BS, Kappes MM, Krupke R, Hennrich F. Separation of single-walled carbon nanotubes by 1-dodecanol-mediated size-exclusion chromatography. ACS Nano 2013;7(4):3557-3564.

32. Kowalczyk B, Lagzi I, Grzybowski BA. Nanoseparations: strategies for size and/or shape-selective purification of nanoparticles. Curr Opin Colloid Interface Sci 2011;16(2):135-148.

33. Wahlund KG. Flow field-flow fractionation: critical overview. J Chromatogr A 2013; 1287:97-112.

34. Peters R, ten Dam G, Bouwmeester H, Helsper H, Allmaier G, vd Kammer F, et al. Identification and characterization of organic nanoparticles in food. Trends Analyt Chem 2011;30(1):100-112.

35. Degueldre C, Favarger PY. Colloid analysis by single particle inductively coupled plasma-mass spectroscopy: a feasibility study. Colloids Surf A Physicochem Eng Asp 2003;217(1-3):137-142.

36. Degueldre C, Favarger PY. Thorium colloid analysis by single particle inductively coupled plasma-mass spectrometry. Talanta 2004;62(5):1051-1054.

37. Pace HE, Rogers NJ, Jarolimek C, Coleman VA, Higgins CP, Ranville JF. Determining transport efficiency for the purpose of counting and sizing nanoparticles via single particle inductively coupled plasma mass spectrometry. Anal Chem 2011;83(24):9361-9369.

38. Reed RB, Higgins CP, Westerhoff P, Tadjiki S, Ranville JF. Overcoming challenges in analysis of polydisperse metal-containing nanoparticles by single particle inductively coupled plasma mass spectrometry. J Anal At Spectrom 2012;27(7):1093-1100.

39. International Organization for Standardization. Nanoparticles: detection and characterisation using single-particle ICP-MS (ISO/ TC 229/SC, N1141); 2013 [cited 2014 Oct 10]. Available from: http://www.iso.org/iso/home/store/catalogue_tc/catalogue tc_browse.htm?commid=381983.

40. Gray EP, Coleman JG, Bednar AJ, Kennedy AJ, Ranville JF, Higgins CP. Extraction and analysis of silver and gold nanoparticles from biological tissues using single particle inductively coupled plasma mass spectrometry. Environ Sci Technol 2013;47(24): 14315-14323. 